\title{
A Method of Component Extraction of EDS and EELS maps
}

\author{
Shixin Wang \\ Micron Technology, Inc., 8000 S. Federal Way, Boise, ID 83707, USA
}

In microanalysis, we generate elemental maps from analytical data, such as EDS and EELS, to show the spatial distribution of elements of interest. In either EDS or EELS, the intensity of a map pixel may have contributions from multiple components. For example, overlapping signals, x-ray fluorescence (for EDS), or spatial mixture can form mixture maps. To obtain a map for each individual component, we need to find a way to calculate each individual contribution. A commonly used method to separate overlapping signals is to deconvolute the source signal. For situation of $\mathrm{x}$-ray fluorescence or spatial mixture, spectral deconvolution is not applicable.

Here we demonstrate a component extraction method on EDS and EELS maps. Let $I(x, y)$ be the intensity function of a map. Suppose $I(x, y)$ has contributions from two components, $A(x, y)$, and $B(x, y)$, from signal $a$ and $b$, respectively. Mapping is obtained through integration of a signal over a fixed window and integration is a linear operation, we thus have, $I(x, y)=A(x, y)+B(x, y)$. If we know $B(x, y)$, we get a pure $A(x, y)$ through a simple subtraction, $I(x, y)-B(x, y)$. The way to find $B(x, y)$ is to form a map $C(x, y)$ over the same region. $C(x, y)$ is a map generated from signal $c$. The criteria of choosing $c$ are: (1) the map $C(x, y)$ has a region free of contribution of $a$, (2) $c$ has no signal overlap with $a$, and (3) the intensity of $c$ is linearly proportional to $b$. With $C(x, y)$, we can strip the contribution of $b$, and obtaining the $A(x, y)$ through the following equation,

$A(x, y)=I(x, y)-k \cdot C(x, y)$

Where, $k$ is a constant independent of spatial coordinates and its value is $B(x, y) / C(x, y)$. $A(x, y)$ can be explicably solved since $I(x, y)$ and $C(x, y)$ are simply the pixel reading of maps. The key is finding the constant $k$. This is through the region free of contribution of $a$, in which $B(x, y) / C(x, y)$ is a constant. By comparing the pixel intensity ratio, we compute the value $k$. With the value $k$ being a constant, eq. (1) is simply image subtraction.

For EDS or EELS, it is always possible to find such a component like c. For example, Fig. 1 (A) is "Si-k $\alpha$ map". Because $S i-k \alpha$ peak overlaps with $W-M \alpha 1$ peak, the map contains $W$ component. From examination of local EDS spectrum, we know the vertical bright structure is pure $W$. Thus we pick a $W$ - $L \alpha 1$ signal to be our $c$ (Fig. 1B). The ratio $k$ is found by comparing pixel intensities of $W-L \alpha 1$ map and "Si-k $\alpha$ map" at a Si-free region (such as the area marked by the box in Fig. 1(B). If the component $c$ is not readily available on the sample, we can put a piece of material containing $c$ onto the sample by FIB manipulation. There are two methods in choosing $c$ : (1) $c$ can be a different signal, such as different EDS peaks, EELS edges, or from different type of detector, of the same element; (2) $c$ can be from a different element of a chemical compound.

The process described by Eq. 1 can be readily extended to maps with more than two components. Fig. 2 shows the result of pure component maps involving signal overlaps and $\mathrm{x}$-ray fluorescence. With iterative application of Eq. 1, a clean spatial distribution of each individual element is obtained (Fig. 2B).

Through computer programming, the calculation of Eq. 1 can be easily automated. Fig. 3 shows a program written with Gatan ${ }^{\circledR}$ DigitalMicrograph scripting language. When a user selects a region for $c$, a subtraction image is formed by $I-k \cdot C$, with $k$ value selected such that the average intensity of the same selected area in the subtraction image is zero. The application of this method is limited to thin samples, when $b: c$ linearity requirement is satisfied. For thick sample, this method losses its accuracy and eventually becomes not applicable when the linearity between $b$ and $c$ does not hold due to absorption. 

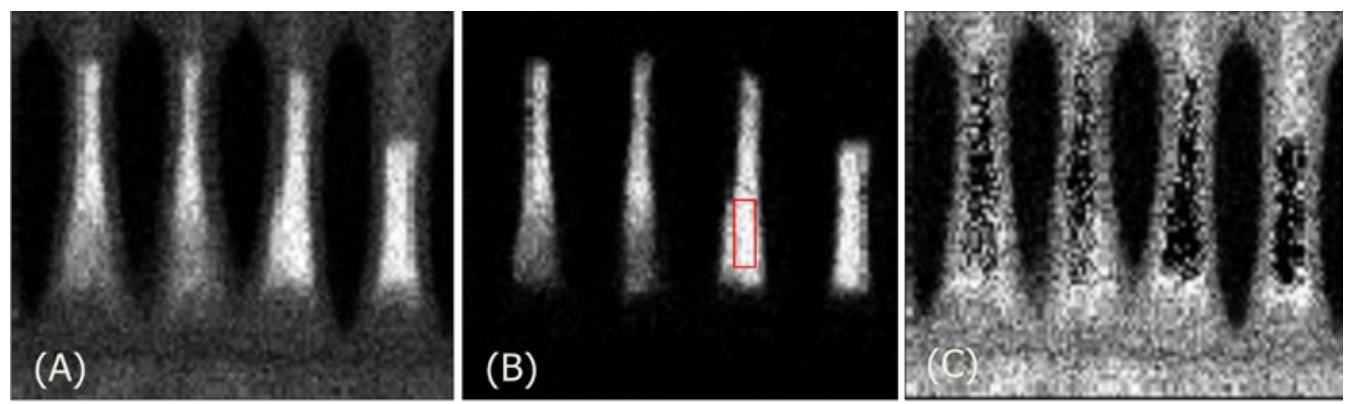

Fig. 1. EDS maps. (A) Si-K $\alpha$ map, contaminated by W-M $\alpha 1$ signal. (B) W-L $\alpha 1$ map. (C) Si map stripped of W component.
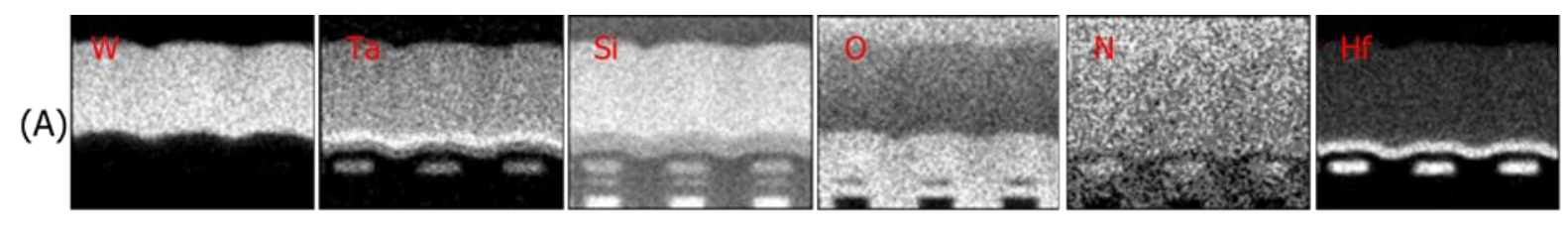

(B)
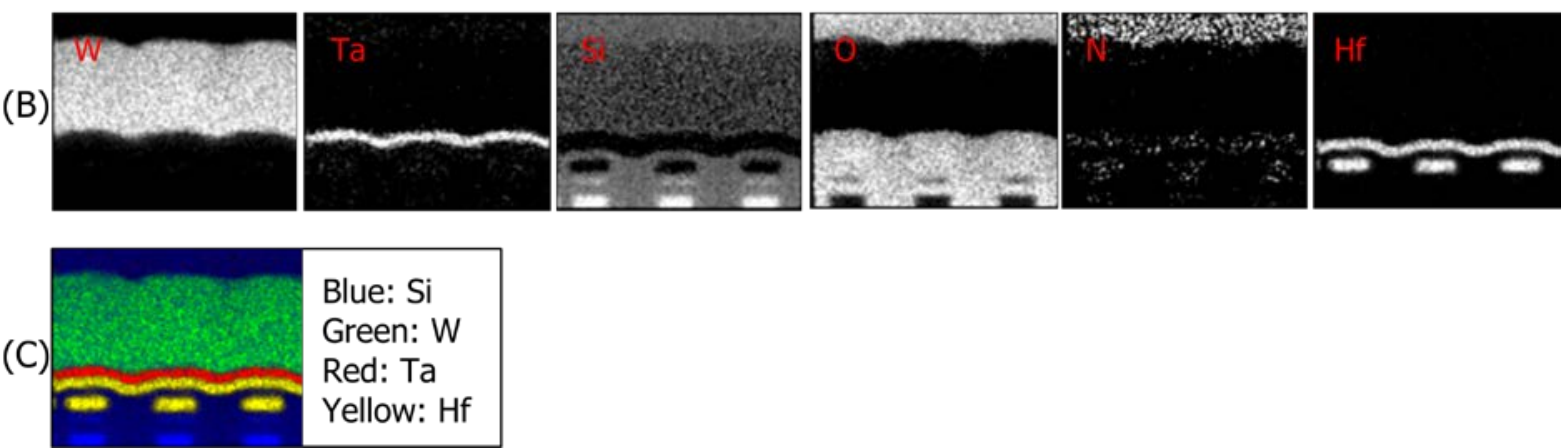

Fig. 2. EDS maps. (A) Original unprocessed maps. (B) pure component maps. (C) color overlays of true elemental maps.

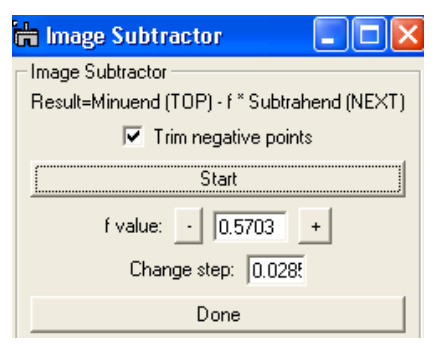

Fig. 3. A computer program to carry out the map component extractions interactively. 